\section{The influence of light deprivation on implantation in the rat}

\author{
SARA C. GILMAN and GLORIA J. FISCHER \\ Departments of Veterinary Physiology and Pharmacology and Psychology \\ Washington State University, Pullman, Wash. 99163
}

The normal pattern of blastocyst implantation in the rat is dependent on the so-called prenidation estrogen surge. Suppression of luteinizing hormone (LH) release by the pituitary interferes with this estrogen release and thereby inhibits implantation. In this study, darkness or blinding during pregnancy increased the variability in distribution of implantation sites and reduced the total number. It is suggested that these effects result, in part, from increased pineal secretion that accompanies light deprivation and, to some extent, interferes with $L H$ release. Since inhibition of implantation following light deprivation is not substantial, however, the pineal would appear to play a minor role in neuroendocrine regulation of the implantation of blastocysts.

Blastocyst survival and implantation are regulated primarily by the ovarian hormones, progesterone and estrogen. Progesterone apparently influences blastocyst survival, and estrogen seems essential for implantation (Cochrane \& Meyer, 1957). In the rat, an increase in estrogen secretion by ovarian tissues reportedly occurs on the fourth day of pregnancy, sensitizing the uterus for nidus fixation (Mayer, 1963; Shelesnyak, Kracier, \& Zielmaker, 1963; Yoshinaga, Hawkins, \& Stocker, 1969). This fourth day estrogen surge appears necessary for ova implantation, as removal of the ovaries before the fourth day postcoitus (pc) prevents implantation, even when adequate amounts of progesterone are administered (Nutting \& Meyer, 1963; Shelesnyak \& Kracier, 1963).

Suppression of $L H$ release by the pituitary inhibits the surge of estrogen secretion and results in a delay or derangement of ova implantation (Hayashida \& Young, 1963 MacDonald, Armstrong \& Greep, 1967; Madhwa Raj, Sairam, \& Moudgal, 1968). Products of the pineal gland, particularly melatonin, cause a decrease in pituitary weight and a decrement in $\mathrm{LH}$ secretion in the female rat (Adams, Wan, \& Sohler, 1965; Hoffmann, 1967). Continuous darkness or blinding produces an increase in pineal weight and activity (Wurtman, Axelrod, \& Kelly, 1968). Such findings suggest that light deprivation might enhance the secretion of the pineal, thereby inhibiting the release of $\mathrm{LH}$ by the pituitary and the surge of estrogen prior to implantation. Inhibition of this estrogen surge might result in a reduced number of ova implants and/or a delay in the time of implantation.

The possibility that light deprivation during pregnancy might reduce the number of ova implants was tested in Experiment 1 by performing laparotomies on rats deprived or not deprived of sensory and/or nonsensory light stimulation during pregnancy. The possibility that light deprivation might delay implantation was tested in Experiment 2, using groups treated similarly. This was done by performing laparotomies daily from the fifth day pc until implants were detected in females that had been blinded or not blinded prior to and/or after insemination.

\section{EXPERIMENT 1}

EFFECT OF BLINDING ON THE

\section{NUMBER OF UTERINE IMPLANTS} Method

Virgin female Sprague-Dawley rats, weighing between 180 and $250 \mathrm{~g}$, were used throughout this investigation. They were maintained on standard laboratory chow and water ad lib. The stage of their estrous cycle was determined by microscopic examination of vaginal smears obtained by saline lavage. After completing two regular estrous cycles, each female was assigned to one of four treatment groups: blinded and housed in controlled light (Group 1 , $B+L)$; visually intact and housed in controlled light (Group 2, L); blinded and housed in the dark (Group 3, (Group 4, D). $\mathrm{B}+\mathrm{D})$; and visually intact in the dark
Blinding was effected by removal of both eyes (bilateral orbital enucleation) under ether anesthesia. The eyelids were sutured shut, and $0.2 \mathrm{ml}$ of penicillin was administered intramuscularly. Immediately after blinding, females in Groups B + L and $L$ were housed individually and subjected to $14 \mathrm{~h}( \pm 15 \mathrm{~min})$ of light from two 40-W soft-white fluorescent lamps and $10 \mathrm{~h}( \pm 15 \mathrm{~min})$ of dark per day for 2 weeks. Light intensity in various areas of the cages was $10-50 \mathrm{fc}$. Although the room was not equipped with temperature and humidity controls, measurements at various times showed little deviation from $22^{\circ} \mathrm{C}$ and $40 \%$ relative humidity.

Two weeks after blinding, each female was caged with a male and daily vaginal lavages were initiated. Lavages and animal transfer were performed in an outer dark room which was equipped with a $7.5 \cdot \mathrm{W}$ dark red light source for use with animals in Groups B + D and D. Total time of exposure to the red light throughout the experiment averaged from 2 to $4 \mathrm{~min}$ per animal, as measured by a stopwatch. On the day sperm were detected in the vaginal smear, the male was removed, and that day was considered Day 1 of pregnancy or Day 1 pc. At $\cdot 8$ days pc, all rats were killed with an overdose of ether or sodium pentabarbitol. When unconscious, they were removed to a lighted room where laparotomies were performed and the number of implantation sites counted

Results

The left half of Table 1 shows the average number of implantation sites in the left and right uterus and the total number of embryos for each of the four light treatment groups. The right half of Table 1 shows the average number of implantation sites in the uterus containing the largest and smallest number of sites and the average absolute difference for each group.

A two-way analysis of variance on the number of implantation sites in each uterus indicated that darkness during pregnancy, whether by blinding

Table 1

The Number of Implantation Sites as a Function of the Presence or Absence of Light Reception and Light Stimulation During Pregnancy

\begin{tabular}{|c|c|c|c|c|c|c|c|c|}
\hline & & & & Mean $N$ & per of $i$ & e Implan & ation Site & \\
\hline Treatr & lent & $\mathbf{N}$ & Left & Right & Total & Largest $\dagger$ & Smallest & rence \\
\hline Intact & $\begin{array}{l}\text { Light } \\
\text { Dark }\end{array}$ & $\begin{array}{l}16 \\
18\end{array}$ & $\begin{array}{l}7.19 \\
6.11\end{array}$ & $\begin{array}{l}6.37 \\
6.17\end{array}$ & $\begin{array}{l}13.56 \\
12.28\end{array}$ & $\begin{array}{l}7.87 \\
7.67\end{array}$ & $\begin{array}{l}5.69 \\
4.61\end{array}$ & $\begin{array}{l}2.18 \\
3.05\end{array}$ \\
\hline Blinded & $\begin{array}{l}\text { Light } \\
\text { Dark }\end{array}$ & $\begin{array}{l}16 \\
16\end{array}$ & $\begin{array}{l}6.69 \\
6.75\end{array}$ & $\begin{array}{l}5.56 \\
7.75\end{array}$ & $\begin{array}{l}12.25 \\
14.50\end{array}$ & $\begin{array}{l}8.12 \\
8.87\end{array}$ & $\begin{array}{l}4.12 \\
5.62\end{array}$ & $\begin{array}{l}4.00 \\
3.35\end{array}$ \\
\hline
\end{tabular}

${ }^{*} S_{\bar{x}} \equiv .21 ;$ tmean of the uterine sides having the largest number of implantation sites 
or by the absence of light, did not produce any difference between the number of implantation sites in the left and right uterus. However, there were significant light treatment effects on the total number of embryos $(F=9.02, \quad d f=1,62, \quad p<.01)$. Specifically, either blinding or darkness resulted in fewer embryos, as expected, but these effects were not additive. Instead, females both blinded and placed in the dark during pregnancy had as many embryos as did control females.

Though of peripheral interest, a second two-way analysis of variance was performed on the number of implantation sites in the side of the uterus having the larger and smaller number of embryos. Results showed significant treatment effects on the absolute difference between the number of implantation sites in the two sides $(F=10.88, \quad \mathrm{df}=1,62$, $\mathrm{p}<.01)$. As can be seen from the absolute difference column in the right half of Table 1, the difference was greater in the light-deprivation grouns than it was in the control group. The difference was reliably greater, however, only for the blinded groups taken together, when compared with the control (the average blinded group difference minus control group difference $=1.47$, which is greater than a critical $t=1.39$ at the .05 level of significance). The absolute difference was not reliably greater for the groups in the dark, taken together, when compared with the control group difference.

\section{EXPERIMENT 2}

EFFECT OF BLINDING ON

\section{TIME OF OVA IMPLANTATION} Method

Six sexually mature, female Sprague-Dawley rats were placed with an experienced male. When sperm were detected in the vagina by saline lavage, one-half of these rats were blinded immediately by bilateral orbital enucleation; the other half were left intact. of another six females, three were blinded for 2 weeks prior to breeding; the other three were left intact for 2 weeks and then bred. Beginning on Day 5 pc, laparotomies were performed daily on all 12 animals until uterine implants were detected.

\section{Results}

Laparotomies showed that all six of the blinded females and all six of the intact females had visible implantations by Day 6 pc. Also, the total number of implants was the same as the number found on Day 9 pc. That is, laparotomies performed as late as Day 9 pe did not reveal the addition of any new implantation sites following initial implantation on Day 6 pc Thus, sensory light

deprivation as great as 2 weeks prior to and during the first few days pc did not delay implantation.

\title{
DISCUSSION
}

Present findings suggest that female albino rats normally have one or two (about $13 \%$ ) more implantation sites on one (left or right) side of the uterus than on the other. Sensory light deprivation during pregnancy increased that difference to three or four (about 30\%) more sites on one side than the other. Apparently, then, sensory light deprivation during pregnancy increases the variability in distribution of implantation sites. Sensory light deprivation from darkness or blinding also reduced the number of implantation sites. These findings support the predicted inhibitory involvement of pineal secretions on $L H$ synthesis and release. However, failure to find fewer implantation sites when females were blinded and in darkness during pregnancy is not consistent with such an interpretation. An explanation of the latter finding, as contrasted with the effects found either from blinding or darkness alone, is obscure.

\section{REFERENCES}

ADAMS, W. C., WAN, L., \& SOHLER, A. Effect of melatonin on anterior pituitary luteinizing hormone. Journal of Endocrinology, 1965, 31,295-296.

COCHRANE, R. L., \& MEYER, R. K. Delayed nidation in the rat induced by progestone. Endocrinology, 1957, 96, 155-159.

\section{Activity of blinded rats in tactually simple and complex environments*}

\author{
GERALD W. MORLOCK and MERLE E. MEYER \\ Western Washington State College, Bellingham, Wash. 98225
}

The optimal level of arousal hypothesis was investigated by measuring the general locomotor activity of blinded albino rats in tactually simple and complex environments. Activity, as measured by the number of photobeam disruptions, was significantly less in the tactually complex environment than in the tactually simple environment. The results were interpreted as supporting the optimal level of arousal hypothesis.

Browman (1942) found surgically blinded male and female rats of a 16-generation inbred line to be more active than nonblinded littermates. Blind hooded rats (Glickman, 1958), albino rats (Klein \& Brown, 1969), and $\mathrm{C} 57 \mathrm{BL} / 6 \mathrm{~J}$ mice (Wimer \& Stern, 1964) were more active than nonblinded controls. Increases in activity associated with the loss of vision have been interpreted, by

*This research was supported in part by a grant to Merle E. Meyer from the Bureau for Faculty Research, Western Washington State College. The assistance of Dr. Louis G. Lippman in performing the optic enucleations is gratefully acknowledged.
Glickman and by Klein and Brown, as supporting Hebb's (1955) optimal level of arousal hypothesis.

Recently, Carlton (1968, 1969) suggested that one result of exploration of a novel environment is habituation to the "nonbiologically significant" stimuli contained in that environment, and that habituation requires protracted exposure to the novel stimuli. For example, Corman \& Shafer (1968), using nonblinded hooded rats, found that introduction of a novel stimulus in a familiar open field resulted in an increase in exploratory behavior, and that removal of a familiar stimulus resulted 